\title{
The impact of homocysteine on the risk of coronary artery diseases in individuals with diabetes: a Mendelian randomization study
}

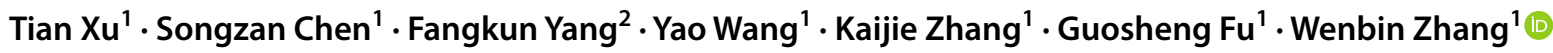

Received: 15 July 2020 / Accepted: 18 September 2020 / Published online: 28 October 2020

(C) The Author(s) 2020

\begin{abstract}
Aims Observational studies have reported that homocysteine (Hcy) is associated with an increased risk of coronary artery disease (CAD) in individuals with diabetes, though controversy remains. The present study aimed to investigate the causal association between Hcy and CAD in individuals with diabetes.

Methods A 2-sample Mendelian randomization (MR) study was designed to infer causality. Genetic summary data on the association of single nucleotide polymorphisms (SNPs) with Hcy were extracted from the hitherto largest genome-wide association study (GWAS) of up to 44,147 individuals of European ancestry. SNP-CAD data were obtained from another recently published GWAS which included 15,666 individuals with diabetes (3,968 CAD cases, 11,696 controls). The fixed-effects inverse variance-weighted method was employed to calculate the effect estimates. Other robust methods and leave-one-out analyses were used in the follow-up sensitivity analyses. Potential pleiotropy was assessed with the MR-Egger intercept test. Results The 2-sample MR analysis suggested no evidence of an association between genetically predicted plasma Hcy levels and CAD risk in individuals with diabetes (odds ratio $=1.14,95 \%$ confidence interval: $0.82-1.58, p=0.43$ ) using 9 SNPs as instrumental variables. Similar results were observed in the follow-up sensitivity analyses. The MR-Egger intercept test indicated no evidence of directional pleiotropy (intercept $=0.03,95 \%$ confidence interval: $-0.08-0.03, p=0.35$ ).

Conclusion This 2-sample MR analysis found no evidence of a causal association between plasma Hcy levels and CAD risk in individuals with diabetes.
\end{abstract}

Keywords Plasma homocysteine levels $\cdot$ Coronary artery disease $\cdot$ Diabetes $\cdot$ Mendelian randomization $\cdot$ Causal association

Managed by Massimo Porta.

Tian $\mathrm{Xu}$ and Songzan Chen have contributed equally to this work

Guosheng Fu

fugs@zju.edu.cn

Wenbin Zhang

3313011@zju.edu.cn

1 Key Laboratory of Biotherapy of Zhejiang Province, Department of Cardiology, Sir Run Run Shaw Hospital, School of Medicine, Zhejiang University, 3 East Qingchun Road, Hangzhou 310016, Zhejiang Province, People's Republic of China

2 Department of Cardiology, Second Affiliated Hospital, School of Medicine, Zhejiang University, Hangzhou 310009, People's Republic of China

\section{Introduction}

Coronary artery disease (CAD) remains the leading cause of death worldwide [1-3]. Despite advances in medical and interventional treatment modalities, outcomes are still unsatisfactory in high-risk subsets of patients [4] and particularly in patients with diabetes. Coronary artery disease is a major prognostic determinant for patients with diabetes as cardiovascular death is responsible for more than $50 \%$ of mortality in patients with diabetes [5]. Therefore, much attention has been paid in the past decades to the identification of new risk factors in order to prevent CAD in individuals with diabetes. Female sex, smoking, obesity, diabetes duration, elevated systolic blood pressure and increased brain-natriuretic peptides can increase cardiovascular risk in people with diabetes [6-9].

Homocysteine (Hcy) is a sulfur-containing amino acid; its metabolism process is dependent on several nutritional 
and genetic factors [10]. High levels of Hcy can lead to an increased state of thrombogenicity, oxidative stress status and endothelial dysfunction [11]. Many studies have found that elevated Hcy levels are a risk factor/biomarker/predictor for developing CAD [12]. However, research on whether elevated levels of plasma Hcy correlate with an increased risk of CAD in individuals with diabetes is still relatively rare. Due to the influence of lifestyles, diet, genetics, and the environment, the incidence of hyperhomocysteinemia has gradually increased [13]. Several studies have shown that plasma Hcy levels in patients with diabetes are increased as compared with those without diabetes [14-16]. Therefore, it is particularly important to determine whether Hcy is causally correlated with an increased risk of CAD in individuals with diabetes.

The Mendelian randomization (MR) approach can effectively overcome some of the limitations of observational studies such as confounding or reverse causation [17]. Mendelian randomization has become increasingly popular for assessing and screening for potentially causal associations [18]. This study aimed to assess the causal association between plasma Hcy levels and CAD risk in individuals with diabetes.

\section{Methods}

\section{Data sources}

The genetic variants associated with Hcy were obtained from the hitherto largest genome-wide association study (GWAS) meta-analysis, with up to 44,147 individuals of European ancestry [19]. The corresponding genetic variants associated with CAD in individuals with diabetes were extracted from a recently published GWAS, including 15,666 individuals with diabetes (3,968 CAD cases and 11,696 controls) [20]. That study was based on UK Bio bank and all the individuals were of European ancestry. Ethics approval was not needed for this current study because it is a secondary analysis of previously published data.

\section{Study design}

A 2-sample MR study was designed to investigate the causal effect of lifetime elevated plasma Hcy levels on the risk of $\mathrm{CAD}$ in individuals with diabetes. The single nucleotide polymorphisms (SNPs) identified as instrumental variables for Hcy had to satisfy the following 3 key assumptions (Fig. 1): (a) SNPs must be strongly associated with plasma Hcy level, (b) SNPs must be independent of confounders, and (c) SNPs must only be associated with the risk of CAD in individuals with diabetes via plasma Hcy level [21].

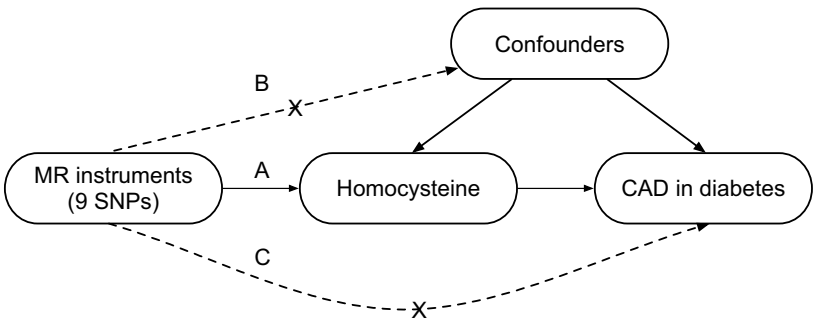

Fig. 1 Three key assumptions of MR study. a SNPs must be associated with Hcy; b SNPs must be independent of confounders; c SNPs should not be directly associated with CAD in patients with diabetes. MR, Mendelian randomization; SNP, single nucleotide polymorphism; Hcy, homocysteine; CAD, coronary artery disease

\section{Selection and validation of SNPs}

First, we selected SNPs associated with plasma Hcy levels at the genome-wide significance threshold $\left(p<5 \times 10^{-8}\right)$ from the Hcy GWAS. Second, we used LD-Link based on European to calculate the pairwise-linkage disequilibrium, evaluating the independence among selected SNPs [22]. When $r^{2}>0.001$, we removed the SNP correlated with more SNPs or with a higher $p$-value. Third, to avoid pleiotropy we looked up the remaining SNPs in the PhenoScanner and excluded those associated with other traits at genome-wide significance [23]. Finally, we calculated the F-statistic to validate the strength of each SNP, which is based on $R^{2}$ (i.e., the proportion of phenotypic variance explained by each SNP).

\section{Primary MR analysis}

In our main analysis, we employed the fixed-effects inverse variance-weighted (IVW) method to evaluate the causal effect of plasma Hcy levels on the risk of CAD in individuals with diabetes. Specifically, we generated a causal estimate for each SNP using the Wald estimator and a corresponding standard error using the Delta method. Subsequently, we obtained the overall estimate by meta-analyzing all the estimates using the fixed-effects IVW method [24].

\section{Sensitivity analysis and pleiotropy assessment}

The random-effects IVW, penalized robust IVW, maximum likelihood, simple median, weighted median, simple mode, weighted mode, MR-Egger, and Mendelian Randomization Pleiotropy Residual Sum and Outlier (MR-PRESSO) methods were employed in follow-up sensitivity analyses. These methods may provide robust estimates against invalid SNPs [25]. In addition, a leave-one-out sensitivity analysis was performed to determine whether the overall estimate was disproportionately affected by a specific SNP. Subsequently, 
the MR-Egger intercept test was conducted to assess the horizontal pleiotropy and a funnel plot was generated to provide a visual inspection. All of the analyses were implemented by the "MendelianRandomization" and "TwoSampleMR" package with R version 3.6.2 (R Core Team 2019).

\section{Results}

\section{SNP selection and validation}

In total, we obtained 18 SNPs associated with plasma Hcy levels at a genome-wide significance level. Among them, 4 SNPs (rs12921383, rs1801133, rs2851391, rs957140) were removed because of linkage disequilibrium and 5 SNPs (rs12921383, rs1801133, rs2851391, rs957140) were excluded due to their associations with other traits at a genome-wide significance level. Finally, the remaining 9 SNPs were identified as instrumental variables in our study. The characteristics of these SNPs and their associations with Hcy and CAD in patients with diabetes are shown in Table 1. All 9 SNPs were valid $(F>10)$.

\section{MR analyses}

The fixed-effects IVW analysis suggested no evidence of an association between genetically predicted plasma Hcy levels and CAD risk in individuals with diabetes (odds ratio $=1.14$, 95\% confidence interval: $0.82-1.58, p=0.43$ ), as shown in Table 2 and Fig. 2a. Similar results were observed in the sensitivity analyses using the other robust methods (Table 2). The leave-one-out analysis and scatter plot also confirmed no evidence of association (Figs. 2b, c). The MR-Egger intercept test indicated no evidence of horizontal pleiotropy (Table 3), which was confirmed by visual inspection of the funnel plot (Fig. 2d).
Table 2 Association of plasma Hcy levels and risk of CAD in patients with diabetes using different methods

\begin{tabular}{lll}
\hline Method & OR $(95 \%$ CI $)$ & $p$ \\
\hline IVW (Fixed-effects) & $1.14(0.82-1.58)$ & 0.43 \\
IVW (Random-effects) & $1.14(0.82-1.58)$ & 0.43 \\
Penalized robust IVW & $1.14(0.81-1.61)$ & 0.46 \\
Maximum likelihood & $1.14(0.82-1.58)$ & 0.43 \\
Simple median & $1.15(0.70-1.87)$ & 0.58 \\
Weighted median & $1.16(0.75-1.79)$ & 0.50 \\
Simple mode & $1.28(0.62-2.63)$ & 0.52 \\
Weighted mode & $1.46(0.71-3.00)$ & 0.33 \\
MR-Egger & $1.70(0.69-4.24)$ & 0.25 \\
MR-PRESSO & $1.14(0.84-1.55)$ & 0.43 \\
\hline
\end{tabular}

Hcy homocysteine, $C A D$ coronary artery disease, $O R$ odds ratio, $C I$ confidence interval, IVW inverse variance-weighted, MR-PRESSO Mendelian Randomization Pleiotropy Residual Sum and Outlier

\section{Discussion}

This is the first 2-sample MR study to investigate the association between plasma Hcy levels and risk of CAD in patients with diabetes. This study found no evidence for the presence of a causal effect of genetically determined plasma Hcy levels on CAD risk in patients with diabetes. Elevated plasma Hcy levels may just be a biomarker for cardiovascular incidents in patients with diabetes. The findings were robust in sensitivity analyses with different statistical models.

Homocysteine, a non-protein amino acid produced by the breakdown of methionine in vivo, has been well known as far back to the 1960s [26]. Disruption of Hcy metabolism leads to hyperhomocysteinemia, which affects multiple body systems and different pathological conditions, including vascular and neurodegenerative diseases

Table 1 The characteristics of 9 SNPs and their associations with Hcy and CAD in patients with diabetes

\begin{tabular}{|c|c|c|c|c|c|c|c|c|c|c|c|c|}
\hline \multirow[t]{2}{*}{ SNP } & \multirow[t]{2}{*}{ Nearest gene } & \multirow[t]{2}{*}{ Chr } & \multirow[t]{2}{*}{ EA } & \multirow[t]{2}{*}{$\mathrm{OA}$} & \multirow[t]{2}{*}{ EAF } & \multirow[t]{2}{*}{$F$} & \multicolumn{3}{|c|}{ SNP-Hcy association } & \multicolumn{3}{|c|}{ SNP-CAD in diabetes association } \\
\hline & & & & & & & Beta & SE & $p$-value & Beta & SE & $p$-value \\
\hline rs12134663 & MTHFR & 1 & $\mathrm{C}$ & A & 0.2 & 145 & 0.101 & 0.011 & $2.54 \mathrm{E}-21$ & -0.0176777 & 0.0364898 & 0.62806302 \\
\hline rs12780845 & CUBN & 10 & A & G & 0.65 & 56 & 0.0529 & 0.009 & $7.8 \mathrm{E}-10$ & 0.00729057 & 0.0289842 & 0.80140001 \\
\hline rs 1801222 & CUBN & 10 & A & $\mathrm{G}$ & 0.34 & 41 & 0.0453 & 0.007 & $8.43 \mathrm{E}-10$ & -0.0258042 & 0.0273547 & 0.34551701 \\
\hline rs 2275565 & MTR & 1 & G & $\mathrm{T}$ & 0.79 & 43 & 0.0542 & 0.009 & $1.96 \mathrm{E}-10$ & 0.00873448 & 0.0325477 & 0.788423 \\
\hline rs234709 & CBS & 21 & $\mathrm{C}$ & $\mathrm{T}$ & 0.55 & 113 & 0.0718 & 0.007 & $3.9 \mathrm{E}-24$ & 0.0464321 & 0.0267226 & 0.0822889 \\
\hline rs42648 & GTPB10 & 7 & G & A & 0.6 & 33 & 0.0395 & 0.007 & $1.97 \mathrm{E}-08$ & 0.0168946 & 0.0272545 & 0.535335 \\
\hline rs4660306 & MMACHC & 1 & $\mathrm{~T}$ & $\mathrm{C}$ & 0.33 & 37 & 0.0435 & 0.007 & $2.33 \mathrm{E}-09$ & -0.0366147 & 0.0283157 & 0.19598 \\
\hline rs7130284 & NOX4 & 11 & $\mathrm{C}$ & $\mathrm{T}$ & 0.93 & 89 & 0.1242 & 0.013 & $1.88 \mathrm{E}-20$ & 0.0616853 & 0.0509219 & 0.22575299 \\
\hline rs838133 & FUT2 & 19 & A & $\mathrm{G}$ & 0.45 & 39 & 0.0422 & 0.007 & $7.48 \mathrm{E}-09$ & 0.00416956 & 0.0276868 & 0.88029301 \\
\hline
\end{tabular}

SNP single nucleotide polymorphism, $H c y$ homocysteine, $C A D$ coronary artery disease, $C h r$ chromosome, $E A$ effect allele, $O A$ other allele, $E A F$ frequency of effect allele, $S E$ standard error 
A
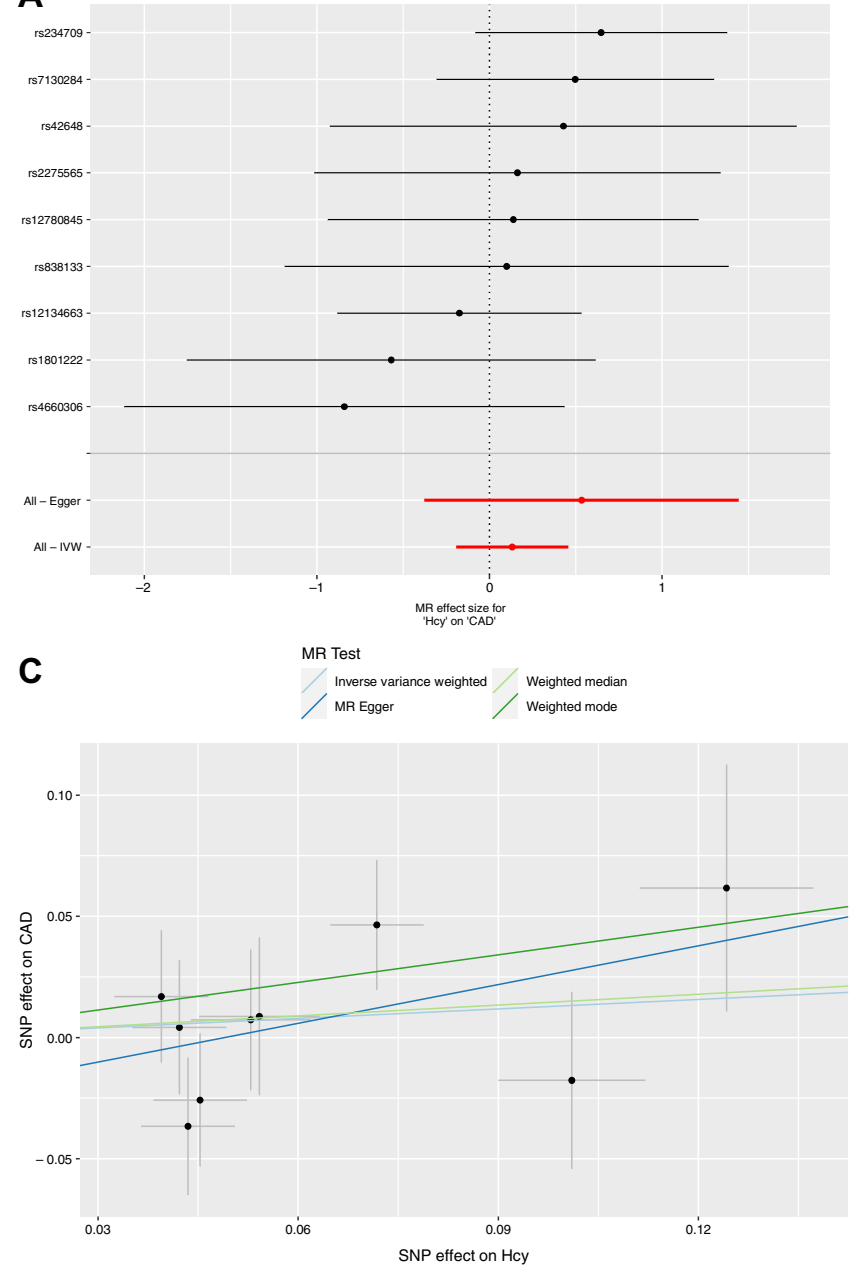

Fig. 2 Forest plot, leave-one-out sensitivity analysis, scatter plot, and funnel plot of the association of plasma Hcy level with the risk of CAD in patients with diabetes. a Forest plot: the dot and bar indicate the causal estimate of plasma Hcy level on risk of CAD in patients with diabetes. b Leave-one-out sensitivity analysis: the dot and bar indicate the estimates and 95\% confidence interval when the specific SNP is removed. c Scatter plot: each black dot indicates a SNP, plot-

Table 3 MR-Egger regression intercept

\begin{tabular}{llll}
\hline Exposure & Outcome & Intercept $(95 \% \mathrm{CI})$ & $p$-value \\
\hline Hcy & CAD in diabetes & $-0.03(-0.08,0.03)$ & 0.35 \\
\hline
\end{tabular}

$M R$ Mendelian randomization, $C I$ confidence interval, Hcy homocysteine, $C A D$ coronary artery disease

The insignificant result $(p>0.05)$ indicates no pleiotropy exists

[27-29]. A direct association between plasma Hcy levels and CAD has been found in observational epidemiological studies [12]. Homocysteine could produce complex changes within the blood vessel wall [30] and may cause CAD through the following 5 pathways [31-33]: (a)
B

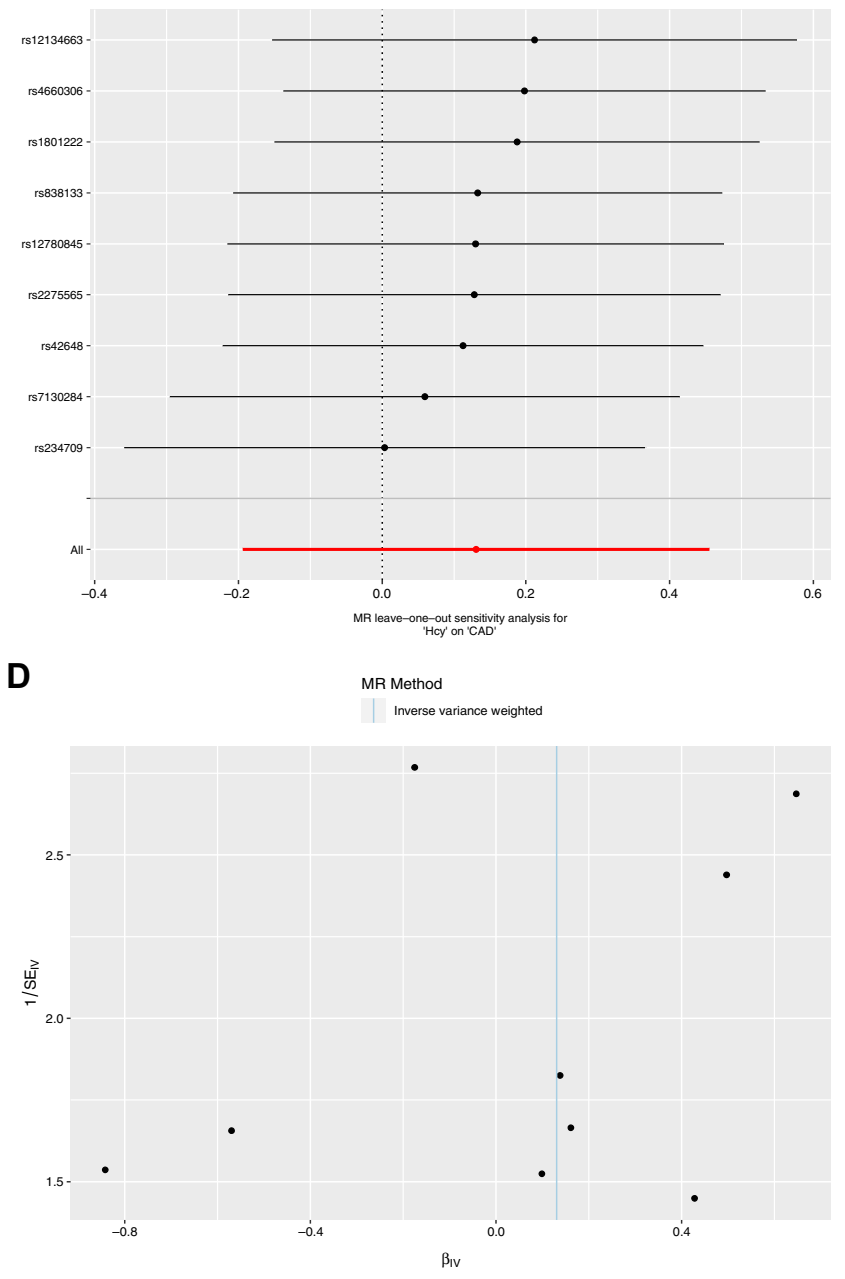

ted by the estimate of SNP on plasma Hcy level and the estimate of SNP on the risk of CAD in diabetes individuals with standard error bars. The slopes of the lines correspond to causal estimates using each of the different methods. d Funnel plot: Each black dot indicates a SNP, and the line indicates the overall estimate using IVW method. Hcy, homocysteine; CAD, coronary artery disease; SNP, singlenucleotide polymorphism; IVW, inverse variance-weighted

vascular endothelial cell damage and dysfunction; (b) dyslipidemia; (c) promotion of the expression of inflammatory factors (e.g., the expression of tumor necrosis factor- $\alpha$ and inducible nitric oxide (NO) synthase (iNOS)); (d) impaired endothelium-mediated platelet inhibition, which enhances coagulation function and induces thrombosis; and (e) stimulation of vascular smooth muscle cell proliferation. Even mild or moderate elevation of plasma concentrations of Hcy (plasma Hcy $>16 \mu \mathrm{M}$ ) can affect coronary and peripheral arteries, eliciting vasomotor dysfunction and increased thrombosis, and consequently increased morbidity and mortality [10, 32].

Some studies have specifically investigated the association between Hcy and atherosclerosis among patients with 
diabetes. A prospective cohort study demonstrated that elevated levels of Hcy are associated with increased cardiovascular risk, especially in patients with diabetes [34]. A larger case-control study found that patients with diabetes and CAD had significantly higher plasma levels of Hcy than patients with diabetes and without cardiovascular damage [35]. However, controversial or negative results have been observed in other studies [36, 37]. In a clinical trial, researchers dosed Hcy in 155 subjects with type 2 diabetes and assessed whether high levels were related to chronic complications. The researchers reported that elevation of plasma Hcy levels in patients with type 2 diabetes was associated with a higher prevalence of peripheral arteriopathy; however, the study found no evidence to support the presence of a relation between Hcy and CAD [38]. Previous findings of an association between high levels of Hcy and increased incidence of CAD in patients with diabetes have been controversial. One possible explanation is that the studies' observational designs mean sample sizes that might not be large enough to detect true associations. Moreover, in observational studies, when confounders are unobserved because they are usually unknown or unmeasured, or when the number of confounders is too large, regression methods may fail to provide unbiased estimates of the true association.

Mendelian randomization is a strategy used to determine whether a biomarker is causally involved in disease development [21]. Other studies have described MR in detail [39, 40]. Briefly, if genetic variants that robustly predict an exposure of interest can be identified, they can be used as unconfounded proxies for that exposure. Associations between the variants and the outcome of interest can thus provide evidence of causation while eliminating the problems of confounding or reverse causation. Genetic association studies are deemed as more similar to randomized clinical trials than other types of observational epidemiological studies due to MR (Mendel's second law) [41]. Multiple GWAS have been published over the past decades, making MR a time- and cost-efficient approach [18].

In this study, we analyzed the association between plasma Hcy levels and CAD in patients with diabetes with the aid of the hitherto largest GWAS meta-analysis. We found that an increase in plasma Hcy levels did not directly lead to the occurrence of CAD in patients with diabetes. A recent study by Liu et al. [42] used the MR approach and, consistent with our findings, reported no causal relation between plasma Hcy levels and coronary heart disease or acute myocardial infarction among the general population. A previous study suggested that high levels of Hcy were related to a higher risk of stroke among general population according to the evidence on MR [43]. However, another recent study used the MR approach to confirm that the causal relation between plasma Hcy levels and stroke is limited to the small vessel stroke subtype within the general population. They found no causal relations between plasma Hcy levels and other stroke subtypes within the general population [44]. The possibility of Hcy being an independent risk factor for CAD in individuals with diabetes was unlikely. But Hcy could potentially participate in the pathogenesis of CAD in patients with diabetes. Elevated plasma Hcy levels may likely be considered biomarkers or concomitants of incident CAD in patients with diabetes. In addition, high Hcy levels observed in traditional observational studies might also be a consequence of CAD that occurred in patients with diabetes.

A strength of this study is its design (i.e., MR analysis of Hcy-related SNPs and SNPs-CAD in individuals with diabetes from large-scale GWAS). Using the 2-sample MR approach, we were able to investigate the effect of Hcy in a large sample size $(44,147$ individuals associated with Hcy, 3968 CAD cases, and 11,696 controls). The following potential limitations warrant discussion. First, it is difficult to completely exclude the influence of potential directional pleiotropy; namely, a genetic variant might affect the outcome via other pathways, which may lead to biased estimates. However, we ruled out as many SNPs associated with recognized confounders as possible. In addition, no evidence of a pleiotropic effect was observed in the MR-Egger intercept test, and similar results were observed in sensitivity analyses using several other models. Second, we were unable to perform a reverse analysis, because the GWAS for Hcy was not publicly available. Third, we only investigated the relation between Hcy and CAD that occurred in patients with diabetes from a genetic point of view, without considering potential environmental factors. Finally, the examined GWAS were primarily conducted in individuals of European ancestry, so the results cannot be generalized to all populations.

\section{Conclusion}

This 2-sample MR analysis found no evidence to support the presence of a causal association between plasma Hcy levels and CAD risk in individuals with diabetes. It is plausible that simply prescribing Hcy-lowering vitamin supplementation may not decrease the incidence of CAD in patients with diabetes in clinical practice.

\section{Availability of data and material}

The datasets analyzed in this study are publicly available summary statistics. 
Acknowledgements The authors would like to express their gratitude to EditSprings (https://www.editsprings.com/) for the expert linguistic services provided.

Author contributions TX and WZ designed the study. TX, SC and FY conducted the analysis and drafted the first draft. YW, KZ and GF reviewed the design and analysis, provided feedback on manuscript drafts, and provided subject matter expertise. All authors reviewed the final version for intellectual content.

Funding This study was supported by Zhejiang Natural Science Foundation (LY18H020007).

\section{Compliance with ethical standards}

Conflict of interest The authors declare no competing interests.

Ethical approval Ethical approval was not needed for this current study because it is a secondary analysis of previously published data.

Informed consent For this type of article, informed consent is not required.

Open Access This article is licensed under a Creative Commons Attribution 4.0 International License, which permits use, sharing, adaptation, distribution and reproduction in any medium or format, as long as you give appropriate credit to the original author(s) and the source, provide a link to the Creative Commons licence, and indicate if changes were made. The images or other third party material in this article are included in the article's Creative Commons licence, unless indicated otherwise in a credit line to the material. If material is not included in the article's Creative Commons licence and your intended use is not permitted by statutory regulation or exceeds the permitted use, you will need to obtain permission directly from the copyright holder. To view a copy of this licence, visit http://creativecommons.org/licenses/by/4.0/.

\section{References}

1. Benjamin EJ, Blaha MJ, Chiuve SE et al (2017) Heart disease and stroke statistics-2017 update: a report from the American heart association. Circulation 135(10):e146-e603

2. Dalen M, Ivert T, Holzmann MJ, Sartipy U (2015) Coronary artery bypass grafting in patients 50 years or younger: a Swedish nationwide cohort study. Circulation 131(20):1748-1754

3. Sappa R, Grillo MT, Cinquetti M et al (2017) Short and longterm outcome in very old patients with ST-elevation myocardial infarction after primary percutaneous coronary intervention. Int J Cardiol 249:112-118

4. Borissoff JI, Spronk HM, ten Cate H (2011) The hemostatic system as a modulator of atherosclerosis. N Engl J Med 364(18): 1746-1760

5. Low Wang CC, Hess CN, Hiatt WR, Goldfine AB (2016) Clinical update: cardiovascular disease in diabetes mellitus: atherosclerotic cardiovascular disease and heart failure in type 2 diabetes mellitus-mechanisms, management, and clinical considerations. Circulation 133(24):2459-2502

6. Fisher DP, Johnson E, Haneuse S et al (2018) Association between bariatric surgery and macrovascular disease outcomes in patients with type 2 diabetes and severe obesity. JAMA 320(15):1570-1582
7. Gallino A (2017) The dilemma of detecting silent myocardial ischemia. Int J Cardiol 244:86

8. Wang H, Ba Y, Cai RC, Xing Q (2019) Association between diabetes mellitus and the risk for major cardiovascular outcomes and all-cause mortality in women compared with men: a meta-analysis of prospective cohort studies. BMJ Open 9(7):e024935

9. Prospective studies C, Asia pacific cohort studies C. (2018) Sexspecific relevance of diabetes to occlusive vascular and other mortality: a collaborative meta-analysis of individual data from 980 793 adults from 68 prospective studies. Lancet Diabetes Endocrinol 6(7):538-546

10. Ganguly P, Alam SF (2015) Role of homocysteine in the development of cardiovascular disease. Nutr J 14:6

11. Paneni F, Beckman JA, Creager MA, Cosentino F (2013) Diabetes and vascular disease: pathophysiology, clinical consequences, and medical therapy - part I. Eur Heart J 34(31):2436-2443

12. Li S, Sun L, Qi L et al (2020) Effect of high homocysteine level on the severity of coronary heart disease and prognosis after stent implantation. J Cardiovasc Pharmacol 76(1):101-105

13. Wierzbicki AS (2007) Homocysteine and cardiovascular disease: a review of the evidence. Diabetes Vasc Dis Res 4(2):143-150

14. Gong T, Wang J, Yang M et al (2016) Serum homocysteine level and gestational diabetes mellitus: a meta-analysis. J Diabetes Investig 7(4):622-628

15. Huang T, Ren J, Huang J, Li D (2013) Association of homocysteine with type 2 diabetes: a meta-analysis implementing mendelian randomization approach. BMC Genom 14:867

16. Majumder M, Mollah FH, Hoque M, Ferdous SA (2017) Serum homocysteine and its association with glycemic control in type 2 diabetic patients. Mymensingh Med J 26(4):921-926

17. Latvala A, Ollikainen M (2016) Mendelian randomization in (epi) genetic epidemiology: an effective tool to be handled with care. Genome Biol 17(1):156

18. Sekula P, Del Greco MF, Pattaro C, Kottgen A (2016) Mendelian randomization as an approach to assess causality using observational data. J Am Soc Nephrol 27(11):3253-3265

19. van Meurs JB, Pare G, Schwartz SM et al (2013) Common genetic loci influencing plasma homocysteine concentrations and their effect on risk of coronary artery disease. Am J Clin Nutr 98(3):668-676

20. Fall T, Gustafsson S, Orho-Melander M, Ingelsson E (2018) Genome-wide association study of coronary artery disease among individuals with diabetes: the UK Biobank. Diabetologia 61(10):2174-2179

21. Emdin CA, Khera AV, Kathiresan S (2017) Mendelian randomization. JAMA 318(19):1925-1926

22. Machiela MJ, Chanock SJ (2015) LDlink: a web-based application for exploring population-specific haplotype structure and linking correlated alleles of possible functional variants. Bioinformatics 31(21):3555-3557

23. Staley JR, Blackshaw J, Kamat MA et al (2016) PhenoScanner: a database of human genotype-phenotype associations. Bioinformatics 32(20):3207-3209

24. Burgess S, Butterworth A, Thompson SG (2013) Mendelian randomization analysis with multiple genetic variants using summarized data. Genet Epidemiol 37(7):658-665

25. Bowden J, Davey Smith G, Haycock PC, Burgess S (2016) Consistent estimation in mendelian randomization with some invalid instruments using a weighted median estimator. Genet Epidemiol 40(4):304-314

26. Caldeira-Araujo H, Ramos R, Florindo C, Rivera I, Castro R, Tavares de Almeida I (2019) Homocysteine metabolism in children and adolescents: influence of age on plasma biomarkers and correspondent genotype interactions. Nutrients 11(3):646 
27. Blom HJ, Smulders Y (2011) Overview of homocysteine and folate metabolism: with special references to cardiovascular disease and neural tube defects. J Inherit Metab Dis 34(1):75-81

28. Doets EL, Ueland PM, Tell GS et al (2014) Interactions between plasma concentrations of folate and markers of vitamin $B_{12}$ status with cognitive performance in elderly people not exposed to folic acid fortification: the Hordaland health study. Br J Nutr 111(6):1085-1095

29. Handy DE, Castro R, Loscalzo J (2011) Epigenetic modifications: basic mechanisms and role in cardiovascular disease. Circulation 123(19):2145-2156

30. Faraci FM, Lentz SR (2004) Hyperhomocysteinemia, oxidative stress, and cerebral vascular dysfunction. Stroke 35(2):345-347

31. Liu B, Chen Z, Dong X, Qin G (2018) Association of prehypertension and hyperhomocysteinemia with subclinical atherosclerosis in asymptomatic Chinese: a cross-sectional study. BMJ Open 8(3):e019829

32. Marti-Carvajal AJ, Sola I, Lathyris D, Dayer M (2017) Homocysteine-lowering interventions for preventing cardiovascular events. Cochrane Database Syst Rev 8(8):CD006612

33. Zhao J, Chen H, Liu N et al (2017) Role of hyperhomocysteinemia and hyperuricemia in pathogenesis of atherosclerosis. J Stroke Cerebrovasc Dis 26(12):2695-2699

34. Ndrepepa G, Kastrati A, Braun S et al (2006) A prospective cohort study of predictive value of homocysteine in patients with type 2 diabetes and coronary artery disease. Clin Chim Acta 373(1-2):70-76

35. Araki A, Sako Y, Ito H (1993) Plasma homocysteine concentrations in Japanese patients with non-insulin-dependent diabetes mellitus: effect of parenteral methylcobalamin treatment. Atherosclerosis 103(2):149-157

36. Krajnc M, Pecovnik Balon B, Krajnc I (2019) Non-traditional risk factors for coronary calcification and its progression in patients with type 2 diabetes: the impact of postprandial glycemia and fetuin-A. J Int Med Res 47(2):846-858
37. Schaffer A, Verdoia M, Barbieri L, Cassetti E, Suryapranata H, De Luca G (2016) Impact of diabetes on homocysteine levels and its relationship with coronary artery disease: a single-centre cohort study. Ann Nutr Metab 68(3):180-188

38. de Luis DA, Fernandez N, Arranz ML, Aller R, Izaola O, Romero E (2005) Total homocysteine levels relation with chronic complications of diabetes, body composition, and other cardiovascular risk factors in a population of patients with diabetes mellitus type 2. J Diabetes Complicat 19(1):42-46

39. Burgess S, Small DS, Thompson SG (2017) A review of instrumental variable estimators for mendelian randomization. Stat Methods Med Res 26(5):2333-2355

40. Kawashiri MA, Tada H, Nomura A, Yamagishi M (2018) Mendelian randomization: its impact on cardiovascular disease. J Cardiol 72(4):307-313

41. Salanti G, Sanderson S, Higgins JP (2005) Obstacles and opportunities in meta-analysis of genetic association studies. Genet Med $7(1): 13-20$

42. Miao L, Deng GX, Yin RX et al (2019) No causal effects of plasma homocysteine levels on the risk of coronary heart disease or acute myocardial infarction: A Mendelian randomization study. Eur J Prev Cardiol. https://doi.org/10.1177/2047487319894679

43. Casas JP, Bautista LE, Smeeth L, Sharma P, Hingorani AD (2005) Homocysteine and stroke: evidence on a causal link from mendelian randomisation. Lancet 365(9455):224-232

44. Larsson SC, Traylor M, Markus HS (2019) Homocysteine and small vessel stroke: A mendelian randomization analysis. Ann Neurol 85(4):495-501

Publisher's Note Springer Nature remains neutral with regard to jurisdictional claims in published maps and institutional affiliations. 